\title{
Survival after first presentation with endomyocardial fibrosis
}

\author{
P. G. D'Arbela, T. Mutazindwa, ${ }^{1}$ A. K. Patel, and K. Somers \\ From The Cardiology Section, Department of Medicine, Makerere University Medical School, \\ Kampala, Uganda
}

Forty-six cases of necropsy endomyocardial fibrosis are analysed and presented with the object of determining their prognosis after first symptoms of the disease. Survival from first symptoms ranged from 12 days to 12 years and 2 weeks, the mean survival being 24 months. The commonest mode of termination was by progressive myocardial failure, very frequently associated with acute respiratory events - either pulmonary oedema, bronchopneumonia, or pulmonary infarction. Unexplained sudden deterioration and collapse probably due to a terminal acute dysrhythmia was the second most important immediate cause of death. Patients with sole left-sided endomyocardial fibrosis run a higher risk of succumbing to acute pulmonary oedema.

Protection by development of severe right-sided endomyocardial fibrosis, organic tricuspid regurgitation, or pulmonary hypertension was therefore a relative advantage, and if other unexpected complications were excluded, survival time was increased. The role of the continuing myocardial destructive process in determining survival rate is discussed.

The anatomical patterns of endomyocardial fibrosis have been well described by Connor et al. (1967, 1968). The clinicopathological features of the disease have also been delineated by Somers et al. (1968a) and Somers, Brenton, and Sood (1968b). Latterly, increasing information has been accumulated on the finer clinical characteristics of the disease, the definition of which has been made possible by the availability of techniques of cardiac catheterization and selective angiocardiography (Somers, D'Arbela, and Patel, 1972). To date there is a paucity of information on the aetiological factors in the disease, and the prognosis in cases in which a diagnosis ' has been reached.

The object of the present study was to determine the survival of cases of endomyocardial fibrosis, from the time of onset of symptoms referable to the disease to the time of death, and to outline possible determinants of duration of survival, and the immediate - cause of death.

\section{Material and methods}

Available case records of 46 patients with endomyocardial fibrosis, adequately studied in life over

Received 2 July 197 I.

1 Supported by the Ford Foundation Vacation Em- ployment Scheme, Makerere University College, Kampala, Uganda.
Io years (1959 to I969), who eventually died and came to necropsy were analysed. The age, sex, tribe, duration of symptoms at the first presentation with the disease, and duration of the disease at death were determined. The duration of symptoms at the first visit was presumed to be the same as the duration of the disease before that visit. The anatomical localization of the lesions of endomyocardial fibrosis was obtained from the pathologist's necropsy reports and was correlated with the clinical presentation. The immediate cause of death was determined from the final notes of the clinician, and the findings at necropsy. An endeavour was made to work out an explanation for the rapid course of the disease in some of the cases and for the relatively longer course in others.

\section{Results}

The age distribution of the 46 cases (Fig. I) showed the usual pattern of involvement of young age-groups (D'Arbela, Kanyerezi, and Tulloch, 1966; Shaper, Hutt, and Coles, 1968). The sex distribution was nearly equal (26 male, 20 female). The tribal distribution conformed to the experience of necropsy endomyocardial fibrosis cases (D'Arbela et al., 1966; Connor et al., 1967, 1968; Shaper et al., 1968), $32(70 \%)$ of the 46 cases being drawn from the Rwanda/Burundi tribal groups.

The duration of illness from the onset of symptoms to death is shown in Fig. 2. Most 


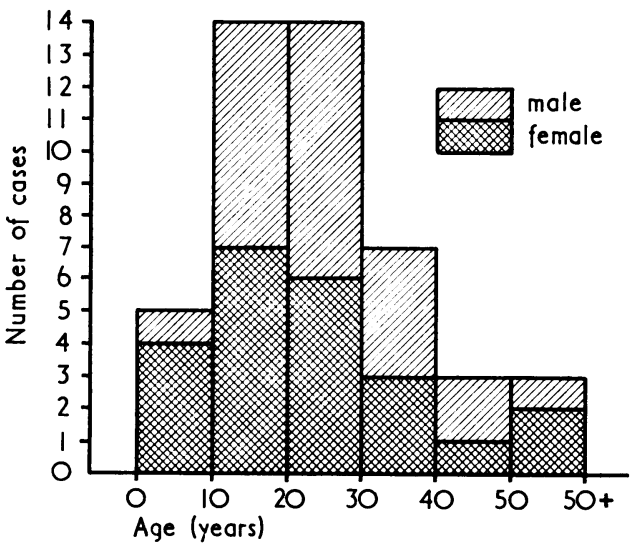

FIG. I Age and sex distribution of 46 necropsy endomyocardial fibrosis cases.

of the patients first presented to hospital soon after the onset of symptoms; a few took much longer, the maximum period recorded in one patient being I I years. Similarly, it is clear from the diagram that in the majority of the patients the progression of the illness was very rapid. In 20 cases $(43.5 \%)$ it was less than I year, in 18 cases $(39.1 \%)$ it was I to 3 years, and in only 8 cases $(17.4 \%$ ) was it 3 to 12 years (Fig. 3). The shortest survival after the onset of symptoms was 12 days and the longest was 12 years and 2 weeks, the mean survival for the 46 cases being 24 months.

The first symptoms in 44 of the 46 subjects were a varied combination of features indicating onset of cardiac failure, namely, shortness of breath, cough, right upper abdominal pain (due to an enlarged tender liver), swelling of the legs, and ascites. Seven-
TABLE I Causes of death in 46 endomyocardial fibrosis necropsy cases

No. of cases

Acspiratory episode as precipitating cause

Progressive myocardial failure

Sudden deterioration and collapse probably due to dysrhythmia

6

Cerebral catastrophe - haemorrhage

Intractable cardiac failure and hepatic failure

Iatrogenic - perforation of aortic valve cusp with ensuing acute heart failure

Isolated acute pulmonary episode in otherwise controlled cardiac failure

Pericardial tamponade

Cor pulmonale with pulmonary tuberculosis

teen of the 44 cases also had palpitations as an early symptom. Six cases admitted to a history of fever, 5 of them being in the group of 44 with cardiac failure symptoms. In the sixth, a case of chronic lymphatic leukaemia, fever was part of the presentation of the leukaemia; it was associated with sore throat, and there were no cardiac symptoms up to the terminal event of cerebral haemorrhage. The forty-sixth case presented essentially as gangrene of the foot from an arterial embolus, with no preceding cardiac symptoms; death occurred from postoperative hepato-renal failure after amputation of the foot.

A breakdown of the causes of death is shown in Table $I$. The commonest cause of death was progressive cardiac failure (I9 cases), followed by sudden deterioration and collapse, possibly due to adverse rhythm changes ( 9 cases), isolated acute pulmonary events, in the form of pulmonary oedema, infarction, or bronchopneumonia (I I cases),

TABLE 2 Anatomical localization of endomyocardial fibrosis lesions in 46 necropsy endomyocardial cases, and relation to survival

\begin{tabular}{|c|c|c|c|c|c|c|c|c|}
\hline Zone involvement & $\begin{array}{l}\text { Mitral valve } \\
\text { structure }\end{array}$ & $\begin{array}{l}\text { Left vent. } \\
\text { apex }\end{array}$ & $\begin{array}{l}\text { Left vent. } \\
\text { outflow tract }\end{array}$ & $\begin{array}{l}\text { Left vent. } \\
\text { obliteration } \\
\text { (variable) }\end{array}$ & $\begin{array}{l}\text { Left vent. } \\
\text { dilatation and / } \\
\text { or hypertrophy }\end{array}$ & $\begin{array}{l}\text { Tricuspid } \\
\text { valve structure }\end{array}$ & Right vent. & $\begin{array}{c}\text { ape } \\
\text { } \\
\sigma \\
\sigma\end{array}$ \\
\hline $\begin{array}{l}\text { Total series } \\
(46 \text { cases })\end{array}$ & $\begin{array}{l}36 \\
(\mathrm{I})\end{array}$ & $\begin{array}{l}30 \\
(I)\end{array}$ & $\begin{array}{l}14 \\
(\mathrm{I})\end{array}$ & 17 & 4 & $\begin{array}{l}\text { I6 } \\
\text { (3) }\end{array}$ & $\begin{array}{l}33 \\
(8)\end{array}$ & $\bar{D}$ \\
\hline $\begin{array}{l}\text { Short survivors } \\
\text { (8 cases) }\end{array}$ & 6 & 5 & I & 4 & I & I & $\begin{array}{c}4 \\
(I)\end{array}$ & 0 \\
\hline $\begin{array}{l}\text { Medium survivors } \\
\text { (30 cases) }\end{array}$ & $\begin{array}{l}24 \\
(I)\end{array}$ & $\begin{array}{l}19 \\
(1)\end{array}$ & $\begin{array}{l}\text { IO } \\
\text { (I) }\end{array}$ & 8 & 2 & $\begin{array}{l}\text { I3 } \\
\text { (3) }\end{array}$ & $\begin{array}{l}21 \\
(5)\end{array}$ & 움 \\
\hline $\begin{array}{l}\text { Long survivors } \\
\text { (8 cases) }\end{array}$ & 6 & 5 & 3 & 4 & I & 5 & $\begin{array}{c}7 \\
(2)\end{array}$ & $\begin{array}{l}\stackrel{2}{(\mathbb{D}} \\
\stackrel{2}{2}\end{array}$ \\
\hline
\end{tabular}

Short survivors, <3 months; medium survivors, 3-36 months; long survivors, > 36 months survival after firs symptoms.

Figures in parentheses are included in those immediately above them; they signify very mild macroscopical involvement. 


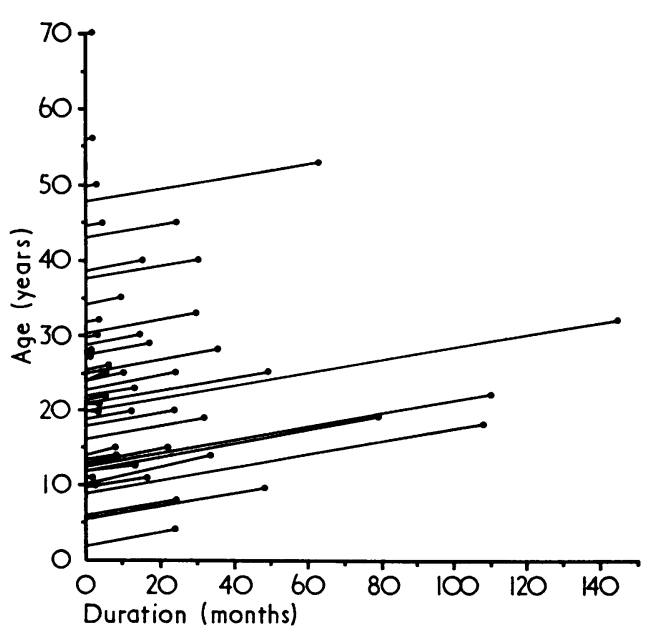

FIG. 2 Age of cases and survival after first symptoms in 46 necropsy endomyocardial fibrosis cases.

cardiac tamponade (I case), hepatic failure ( 3 cases), iatrogenic perforation of the aortic cusp at cardiac catheterization (I case), and cor pulmonale with pulmonary tuberculosis (I case). Overall acute pulmonary episodes precipitated death in 21 cases, acute pulmonary oedema being the most important (I2 cases) followed by pneumonia (IO cases), and pulmonary embolism and/or infarction (4 cases). In a number of cases these varied types of respiratory events occurred together.

The anatomical localization of the endomyocardial fibrosis lesion in the hearts of the 46 necropsy cases is shown in Table 2. Overall assessment of the entire group did not reveal any obvious differences between the cases to explain the difference in survival time.

\begin{tabular}{lll}
\hline $\begin{array}{l}\text { Right vent. } \\
\text { obliteration } \\
\text { (variable) }\end{array}$ & $\begin{array}{l}\text { Right vent. } \\
\text { dilatation and / } \\
\text { or hypertrophy }\end{array}$ & $\begin{array}{l}\text { Clinical } \\
\text { pulmonary } \\
\text { hypertension }\end{array}$ \\
\hline I2 & 17 & 26 \\
I & I & 3 \\
7 & 14 & 17 \\
4 & 2 & 6
\end{tabular}

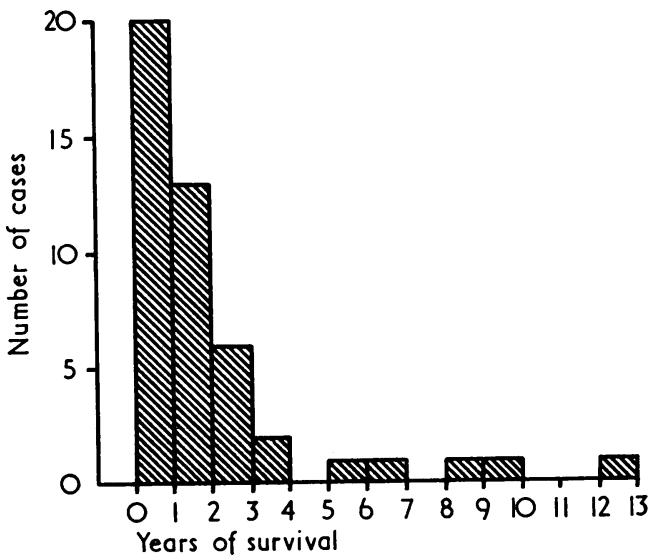

FI G. 3 Number of cases and years of survival after first symptoms in 46 necropsy endomyocardial fibrosis cases.

But when the short survivors (dying within 3 months of onset of illness) and the long survivors (surviving at least 3 years after onset of the symptoms) were compared, certain differences emerged. The series in each group was small for conclusions to be drawn. Generally, however, it was apparent that all the long survivors developed some mechanism or a combination of mechanisms to protect their lungs against overwhelming pulmonary oedema in the presence of left-sided disease - in the form of either severe obliterative right ventricular endomyocardial fibrosis, tricuspid regurgitation, or pulmonary hypertension. On the contrary, few of the short survivors did and most of them succumbed to acute respiratory episodes.

Hence, of the 8 long survivors, 6 had mitral valve posterior leaflet endomyocardial fibrosis, mitral stenosis coexisting in one. In 4 of the 6 cases there was associated partial obliteration of the left ventricular cavity. This, however, did not appear to be prejudicial to long survival, as the third, the fifth, the sixth, and the eighth longest survivors had this feature. The 6 long survivors with mitral regurgitation therefore survived by virtue of developing one or a combination of tricuspid regurgitation resulting from severe right ventricular endomyocardial fibrosis in 3 , combined with predominant tricuspid valve and tricuspid valve papillary muscle implication in 1 , or pulmonary hypertension (in 5 including 2 of the forementioned 3 , and 3 with little or no rightsided diseases). The remaining 2 cases in which there was no mitral valve or left ventricular disease had well-developed severe right ventricular endomyocardial fibrosis (in I), and tricuspid valve and tricuspid valve papillary muscle involvement in both cases. 
It is evident that organic tricuspid regurgitation occurred in 5 of these 8 cases. The mode of death in the whole group of long survivors was progressive myocardial failure in 4, associated with pneumonia in $I$, rapid deterioration and collapse probably referable to a dysrhythmic cause in 2, cardiac tamponade from pericardial effusion combined with advanced myocardial failure from severe involvement in $I$, and peritonitis and terminal bronchopneumonia in $\mathbf{I}$.

Of the 8 short survivors, only I had combined severe left ventricular and severe right ventricular disease with partial obliteration of both ventricles. He died of severe intractable cardiac failure. Of the remaining 7, 4 had only mild (in I) and moderate (in 3 ) right ventricular endomyocardial fibrosis confined to the apex of the ventricle, and 3 did not have rightsided disease at all. Pulmonary hypertension occurred in only 2 of the former 4 , and in one of the latter 3, who survived slightly longer than those without this feature (II weeks as compared with 5 and $5 \frac{1}{2}$ weeks, respectively). None of the 7 cases had organic tricuspid regurgitation, and in the majority of them (5 cases) death was associated with terminal respiratory events, in the form of severe progressive pulmonary oedema (in 4) associated with pulmonary infarction (in I) and mitral stenosis (in another I), and pneumonia in the fifth case. Of the remaining 2 cases, I died from coincidental acute hepatorenal failure after an operation while the other died suddenly, probably from a dysrhythmic cause.

\section{Discussion}

Prognostication in endomyocardial fibrosis cases is difficult unless all the possible number of factors at play are taken into account. Parry and Abrahams (1965) have advanced a suggestion of 'arrested' cases, implying a situation in which the active myocardial destruction and fibrosis occurs at a given time and ceases to progress subsequently. This suggests that in other cases the destructive activity may be continuous. However, at present we have no means of determining the progress of the myocardial destruction. Random studies of enzyme changes also have not given any clue (Campbell and Somers, 1960). Presumably in some cases the activity becomes arrested, and depending on the extent of injury that has been inflicted, survival may be longer than in those in whom the destruction is continuous. The absence of any knowledge of the aetiological factors involved in endomyocardial fibrosis is a further handicap to the clarification of the issue of the nature and course of the active destructive process. But if this is an extraneous factor like an infection, as proposed by Ive et al. (1967), then presumably eradication of the infection and prevention of further exposure or sensitization might improve the survival provided the damage already done was not too extensive.

In the already established disease implicating the left side of the heart, protection of the lungs against pulmonary oedema, by development of severe right ventricular disease or organic tricuspid regurgitation, which would reduce pulmonary flow, or by development of pulmonary hypertension, appears to be a relative advantage, as it usually is, in mitral valve disease of other aetiology or in left-toright shunts. On the whole, taken as isolated entities, predominantly right-sided endomyocardial fibrosis carries a better prognosis than the left-sided disease. However, there are some cases in which presumably the severity of the acute episode or the extent of damage of the myocardium plays a vital role in determining the outcome of the case, just as it would in myocardial infarction.

The high incidence of unexplained sudden deterioration and collapse ( 9 cases) does suggest a strong possibility of a dysrhythmic termination, though no terminal electrocardiographic monitoring was available to confirm this impression.

The relatively high incidence of pneumonia associated with the terminal phase of many of the cases calls for more vigilance in the exclusion of this factor and other acute respiratory episodes in all cases which present with recent unexplained deterioration.

Clinical experience suggests that if the patients presenting with these complications were safely seen through these phases, with the aid of antibiotics in case of pneumonia, their survival time might be increased.

We are grateful to our colleagues in the Department of Pathology for records of necropsy findings and to the British Heart Foundation for the continuing support of our programme in cardiology.

\section{References}

Campbell, J., and Somers, K. (1960). Serum transaminase in endomyocardial fibrosis. British Medical fournal, $x, 1540$.

Connor, D. H., Somers, K., Hutt, M. S. R., Manion, W. C., and D'Arbela, P. G. (1967). Endomyocardial fibrosis in Uganda (Davies' disease) Part I. American Heart fournal, 74, 687.

Connor, D. H., Somers, K., Hutt, M. S. R., Manion, W. C., and D'Arbela, P. G. (1968). Endomyocardial fibrosis in Uganda (Davies' disease) Part II. American Heart fournal, 75, 107. 
D'Arbela, P. G., Kanyerezi, R. B., and Tulloch, J. A. (I966). A study of heart disease in the Mulago Hospital, Kampala, Uganda. Transactions of the Royal Society of Tropical Medicine and Hygiene, 60, 782.

Ive, F. A., Willis, A. J. P., Ikeme, A. C., and Brockington, I. F. (1967). Endomyocardial fibrosis and filaria. Quarterly fournal of Medicine, 36, 495.

Parry, E. H. O., and Abrahams, D. G. (1965). The natural history of endomyocardial fibrosis. Quarterly fournal of Medicine, 34, 383.

Shaper, A. G., Hutt, M. S. R., and Coles, R. M. (1968). Necropsy study of endomyocardial fibrosis and rheumatic heart disease in Uganda 1950-1965. British Heart fournal, 30, 391.

Somers, K., Brenton, D. P., D'Arbela, P. G., Fowler, J. M., Kanyerezi, B. R., and Sood, N. K. (1968a).
Haemodynamic features of severe endomyocardial fibrosis of right ventricle, including comparison with constrictive pericarditis. British Heart fournal, 30, 322.

Somers, K., Brenton, D. P., and Sood, N. K. (1968b). Clinical features of endomyocardial fibrosis of the right ventricle. British Heart fournal, 30, 309.

Somers, K., D'Arbela, P. G., and Patel, A. K. (1972). Endomyocardial fibrosis, a clinical review. In Medicine in a Tropical Environment. British Medical fournal. In the press.

Requests for reprints to Dr. Paul G. D'Arbela, Medical School, Mulago Hospital, P.O. Box 7072, Kampala, Uganda, E. Africa. 